\title{
NECESIDADES, RAZONES, DERECHOS
}

\section{INTRODUCCIÓN. PRESUPUESTOS}

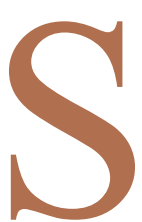

i bien nuestro objetivo es el examen del carácter normativo de las necesidades como punto de partida para la discusión de la posibilidad de fundamentación de los derechos humanos en las necesidades básicas, trataremos de abordar en primer lugar los ineludibles presupuestos de una reflexión como ésta, es decir, el concepto, alcance y función de la noción de necesidades y las teorías sobre las mismas. El intento de elaboración conceptual de las necesidades humanas básicas se ha realizado desde perspectivas teóricas muy diferentes. Todas ellas han de hacer frente a algunos problemas comunes que tienen importantes consecuencias. Nos referiremos a continuación, brevemente, a cinco de esas cuestiones, para detenernos en una sexta -el carácter normativo del concepto de necesidades- que es la que incide más directamente sobre nuestro propósito.

\section{El concepto de necesidad}

Un primer nivel de dificultad deriva de la propia determinación del concepto. Desde todas las perspectivas desde las que se ha estudiado (psicología, sociología, antropología, ciencia política, economía $)^{1}$ se pone de relieve el obstáculo que supone

\footnotetext{
${ }^{1}$ Esta pluralidad de perspectivas exige, aun cuando sea con brevedad, que nos refiramos al proceso de análisis por el que ha pasado la noción de necesidades. En los inicios de la psicología social lo que llamamos necesidades quedaba subsumido en el concepto de instintos, W. McDougal, Introduction to Social Psychology, London, Methen, 1908. Sin embargo, hubo un momento en el que se produjo un viraje hacia nociones tales como deseos, motivos, impulsos, voliciones y en el caso de H. A. Murray a la de necesidades cfr. Explorations in Personality, New York, Oxford U. Press, 1938. Este giro no suponía
} 
determinar ese concepto positivamente, y en consecuencia se acude al criterio de la determinación por la negación. El método que se sigue consiste en examinar la privación continua de una necesidad en términos de sus consecuencias en la integridad somática o psicológica de un individuo, señalando así los efectos fisiológicos, psicológicos, etc., que produce la carencia de «algo», atendiendo, por ejemplo, a las frustraciones, insatisfacciones, estados patológicos, situaciones de carencia material de determinados objetos, etc. ${ }^{2}$

un simple cambio de nomenclatura, sino que trataba de superar el paradigma propio del determinismo biológico al que hacían referencia los instintos, dando paso a una comprensión de las influencias socioculturales en el comportamiento. Por ello, la mayor parte de los análisis sobre necesidades desde esta perspectiva ponen en relación la idea de necesidades con la de motivación y la presentan como esfera explicativa del comportamiento humano. Así, A. H. Maslow, Motivation and Personality, New York, Harper, 1954; De. McDLelland, The Achievement Motive, New York, Appleton, 1953; E. Fromm, The Sane Society, New York, Hott, 1955; R. Peters, The Concept of Motivation, London, Routledge and K. Paul, 1958. El paso de los instintos a las necesidades también se produjo en el campo de la antropología, fundamentalmente por parte de los defensores del relativismo cultural, por ejemplo, M. J. Herkovits, o en las tablas de necesidades elaboradas por B. Malinowski, o las obras de K. Lorenz. Desde la perspectiva de los análisis sociológicos puede verse los escritos, por ejemplo, de A. Etzioni, The Active Society: A Theory of Societal and Political Processes, New York, Free Press, 1968, y «Basic Human Needs, Alienation and Inauthenticity», American Sociological Review, vol. 33, 1968, págs. 870-85. Del mismo modo, desde los análisis de ciencia política hubo una recuperación de la noción de necesidades básicas cuyo impulsor fue Ch. Bay, «Needs, Wants and Political Legitimacy», Canadian Journal of Political Science, vol. 1, 1968, págs. 241-60. S. A. Renshon, Psychological Needs and Political Behaviour, New York, Free Press, 1974, así como el colectivo Human Needs and Politics, Sidney, Pergamon, 1978, dirigido por R. Fitzgerald. Por supuesto, sin olvidar que el arranque de las teorías sobre necesidades encuentra un punto de partida básico en la obra de Marx, del que parten, por ejemplo, los análisis freudo-marxistas, de un lado, el trabajo de Stoyanovitch y Poulantzas, de otro, y una de las construcciones que hoy es posiblemente de las más citadas, la obra de Heller, por ejemplo, La teoría de las necesidades de Marx, Barcelona, Península, 1974, «Can true and false needs be posited?», The Power of Shame, London, Routledge and K. Paul, 1985. En este sentido también, I. Illich, Toward a History of Needs, New York, Pantheon, 1978, o W. Leiss, The limits to Satifaction: an Essay on the problem of needs and comodities, Toronto, Univ. Toronto Press, 1976. Por otra parte, habría que hacer constar también los trabajos que desde perspectivas filosóficas no marxistas hoy son importantes para el análisis de las necesidades. Por ejemplo, P. Springborg, The problem of human needs and the critique of civilisation, London, Allen and Unwin, 1981, o el más reciente de D. Braybrooke, Meeting Needs, New Jersey, Princenton, Univ. Press, 1987.

${ }^{2}$ En este sentido, por ejemplo, Bay define las necesidades como tendencias del comportamiento cuya frustración o cuya negación continuada da lugar a 
Parece, pues, que uno de los criterios básicos y más generales para definir las necesidades es considerar que éstas consisten en una «falta de» o en una «carencia». Sin embargo, resulta posible tratar de definir las necesidades con independencia de que aquí y ahora se carezca de ellas o no. No obstante, aun cuando la necesidad no esté conceptualmente relacionada con una carencia es cierto que a menudo la implica. Es decir, que una forma primaria de entender la necesidad sería independiente de la carencia y una forma secundaria se daría allí donde se describe una necesidad como carencia de aquello que se necesita. ${ }^{3}$ Thomson señala, a estos efectos, que las necesidades pueden ser aquellas situaciones o cosas que necesitamos tanto si carecemos de ellas como si no, pero pueden ser aquellas cosas de las que carecemos y necesitamos. ${ }^{4}$ Desde luego, una de las ideas básicas que se ponen de relieve en la noción de necesidad es la de privación ${ }^{5}$ de aquello que puede ser básico e imprescindible. En este sentido Galtung ${ }^{6}$

respuestas patológicas. «Needs, Wants...», art. cit. pág. 242. Enunciado que recuerda al de Maslow cuando escribe: «Un hombre al que se frustran alguna de sus necesidades básicas puede ser considerado como un enfermo o al menos, como algo inferior a un ser humano completo», Motivation and Personality, cit. pág. 57. Etzioni sostiene que una necesidad es aquella situación por la que a una persona se le niega un tipo específico de experiencia al coste de una tensión intrapersonal. «Basic Human Needs...», art. cit., pág. 871, y esta situación puede ser analizada, entre otros indicadores, a partir de los costes de socialización, así como los costes personales a los que da lugar (págs. 874-76). C. A. Mallmann define las necesidades como «requisitos genéricos que todos los seres humanos tienen en orden a no estar enfermos, por el hecho de ser miembros de la especie humana» «Society, Needs...», Human Needs, cit., pág. 37.

${ }^{3}$ Entre quienes entienden que la necesidad siempre implica una carencia o falta, puede verse, Peters y Hirst, The Logic of Education, London, Routledge and K. Paul, 1970, pág. 33, Wollheim, Nature and Conduct, London, McMillan, 1975, pág. 174, y frente a esta idea, la postura más radical puede estar representada por White, «Needs and Wants», Proceedings of the Philosophy of Education Society of Great Britain, vol. 8, 1974, pág. 164.

${ }^{4}$ G. Thomson, Needs, London, Routledge and K. Paul, 1987, pág. 12.

${ }^{5}$ F. Rosen, «Basic Needs and Justice», Mind, vol. 86, 1977, págs. 88-94.

${ }^{6}$ J. Galtung y A. Wirak, Human Needs, Human Rights and the theories of development, UNESCO, París, 1976, pág. 43. Utilizando el paradigma de la teoría de los sistemas, Sicinski llega a conclusiones parecidas. Define una necesidad como una condición necesaria para el funcionamiento normal de un sistema en su entorno. De donde las necesidades fundamentales serían aquellas cuya no satisfacción tiene como resultado la aniquilación del sistema, siendo las demás necesidades las que como consecuencia de su falta de satisfacción llevan a la incapacidad de sistema para realizar alguna de sus funciones. «The concepts of need and value in the light of the systems approach», Social Science Information, vol. 17, 1978, págs. 73-4. 
señala que existiría una necesidad humana en aquellas situaciones en las que su no satisfacción puede ocasionar una «destrucción, desintegración o no existencia de un ser humano». Esta consideración de las necesidades nos pone directamente en relación con la noción de daño. ${ }^{7}$ A través de esta idea se quiere expresar que la carencia de aquello que se necesita repercute directamente en la calidad de vida humana y que tal perjuicio respecto a algún aspecto de la vida se mantiene a menos que se satisfaga la necesidad en cuestión, no habiendo otro tipo de alternativas, por tanto no es un mero contratiempo pasajero, sino una «degeneración» permanente de la calidad de vida humana que se mantiene en tanto no se obtiene la satisfacción adecuada.

\section{La fundamentación empírica de las necesidades}

En segundo término, la mayor parte de quienes se han ocupado del tema advierten que cualquier teoría sobre necesidades exige una fundamentación empírica y, sin embargo, este requisito constituye al mismo tiempo su mayor dificultad, por lo que la determinación de qué son y cuáles sean las necesidades humanas básicas se realiza en la mayor parte de los casos desde premisas normativas. Esto no significa que no puedan establecerse indicadores o índices en algunos supuestos concretos, por ejemplo, nutrición, salud, educación, aunque, de hecho, las investigaciones empíricas son insuficientes, salvo estos indicadores, entre otras cosas porque, como se ha apuntado, no podemos tener la seguridad de conocer todos los hechos empíricos relevantes y por la propia historicidad de las necesidades. Pero respecto al conjunto de necesidades básicas, en la mayor parte de las propuestas se parte de un paradigma previo de naturaleza humana, o de sociedad sana, de calidad de vida u otros paradigmas

\footnotetext{
${ }^{7} \mathrm{Al}$ respecto, por ejemplo, el planteamiento de Hare, aun cuando sugiere que el concepto de daño ha de entenderse en términos de privación de lo que se desea: Essays on Moral Concepts, London, McMillan, 1972, págs. 97-8. Para la determinación del concepto de necesidades fundamentales en relación con la idea de daño parece preciso superar la concepción de éste sobre la base de los deseos. Resulta preciso también atender al desarrollo conceptual de la noción realizado por Feinberg, por ejemplo, en Social Philosophy, Prentice-Hall, 1973, cap. V y Harm to Others, Oxford Univ. Press, vol. 1, 1987, esp. cap. I. Aun cuando lo más útil a nuestros efectos sería describir la noción de daño para explicar las necesidades y por tanto no explicar el concepto en términos de necesidades.
} 
similares. ${ }^{8}$ A todo ello hay que añadir, además, que, por un lado, las necesidades no aparecen en forma pura, fuera de un contexto social específico, lo que, por cierto, es una -no la única- de las razones por las que resulta difícil admitir el carácter categórico (en sentido fuerte) de las necesidades. Por otro, los valores personales y colectivos afectan directamente a la definición de necesidades y a las formas a través de las que se satisfacen. ${ }^{9}$ Finalmente, las dimensiones de las necesidades son plurales y no permiten que se establezcan medidas cuantitativas con facilidad. Una aproximación a las necesidades, incluso la más cuantitativa, afecta a lo que debería o podría ser. Tras ese cúmulo de dificultades, el problema es, desde luego, dar razón de por qué algo ha de ser realizado o satisfecho en orden a que el sujeto de la necesidad cumpla su función como ser humano. Esto resulta más fácil de explicar a través de las situaciones que impiden el desarrollo que a través de aquéllas lo promueven. Por todo ello, la determinación de las necesidades exige atender conjuntamente a una doble perspectiva que podemos calificar como ontológica e histórica.

\section{Jerarquización y catálogo de las necesidades}

Dificultades de otro orden aparecen también en los intentos de jerarquización y catalogación de necesidades. En este punto vuelven a plantearse los problemas relativos a la determinación empírica y por otro lado las cuestiones en relación con las llamadas necesidades «secundarias». En general, existe una

${ }^{8}$ Respecto a la relevancia del criterio de salud mental véase, O. Klineberg, «Human Needs: a Social-Psichological Approach», Human Needs, cit., págs. 29-32. El concepto de sociedad sana como adaptación de los modelos sociales a las necesidades humanas se encuentra en Fromm, The Sane Society, cit., págs. 72 y 64 y sigs. Cfr. también la definición de Maslow: la sociedad «sana» es aquella que permite que se produzcan o emerjan los propósitos más elevados del hombre porque satisface todas las necesidades básicas. «The instinctoid nature of basic Needs», Journal of Personality, vol. 22, 1954, págs. 328 y sigs. También C. A. Mace «Homeostasis, needs and values», British Journal of Psychology, vol. 44, 1953, págs. 200-210.

${ }^{9}$ Hay algunos intentos de determinar las necesidades clasificándolas de acuerdo con sus correspondientes satisfacciones. Esto es lo que propone, por ejemplo, Y. Fridman, cuyo listado de necesidades puede verse en «About implicit limitations on satisfiers» o la tipología propuesta por C. Mallman, «Society, Needs and Rights: A Systemic Approach», ambos en Human Needs, cit., págs. 40-41 y 155. Sin embargo, las dificultades de determinar las necesidades de este modo son enormes debido a que lo que sí está sujeto al cambio histórico y socio-cultural son, desde luego, las formas de satisfacción de las necesidades. 
identificación entre necesidades primarias y necesidades fisiológicas o biofísicas que son aquellas que deben ser satisfechas para el mantenimiento de la supervivencia. Uno de los principales problemas aparece cuando el modelo de estas necesidades se propone como paradigma de comprensión del resto de las necesidades. Esto no significa que todos los autores acepten la idea de construir una jerarquía de necesidades. Por el contrario, ese intento ha recibido serias críticas, ${ }^{10}$ ya que, hasta el momento, los intentos de ordenación gradual se han formulado en alguna de estas modalidades:

a) Como una tesis axiológica, tratando de mostrar que las necesidades humanas son más elevadas cuanto menos compartidas con los animales.

b) Como una tesis empírica, al tratar de demostrar que las necesidades que se encuentran en la base de la jerarquía son las que se persiguen de hecho por todos los hombres.

c) Como tesis normativa, que trata de poner de relieve que antes de que encuentren satisfacción las necesidades más elevadas deben estar cubiertas las más básicas.

4. Delimitación de las necesidades: deseos, intereses, instintos, aspiraciones, etc.

Existen algunos problemas, a su vez, a la hora de delimitar el concepto de necesidades de otros conceptos afines como deseos, intereses, aspiraciones, impulsos, etc. Resulta preciso, pues, una depuración terminológica en ese sentido. En principio podría decirse que en cuanto criterios de orientación de la conducta las necesidades proporcionarían razones de diferente orden (intuitivamente habría una coincidencia en que se trata de razones de mayor peso) que los deseos, intereses o voliciones, ${ }^{11}$ en sentido contrario, o, al menos, no coincidente. ${ }^{12}$

${ }^{10} \mathrm{Al}$ respecto, J. Galtung, «The Basic Needs Approach», Human Needs, cit., págs. 67-71.

${ }^{11}$ Respecto a las diferencias entre necesidades y voliciones puede verse $\mathrm{Ch}$ : Bay «Needs, Wants...», art. cit., pág. 242, «Human Needs and Political Education», Human Needs and Politics, cit. págs. 25 y sigs. W. Meyer, «Democracy: needs over wants», Political Theory, 1974, Pág. 203. Sadurski, «Genuine Needs», Political Theory, 1983, págs. 421 y sigs. A. White, «Needs and Wants», art. cit. págs. 159-180. S. A. Renshon, «Human Needs...», Human Needs and Politics, cit. págs. 62-4.

${ }^{12}$ N. MacCormick, «Children's Rigths: A test-case for theories of Right», ARSP, 1976, pág. 310. R. Adler, Taking Juvenile Justice Seriously, Edimburgh, Scottish Academic Press, 1985, págs. 65-74. 
Ahora bien, la distinción resulta necesaria, en primer lugar y sobre todo, porque las necesidades no constituyen un acto intencional. No elegimos nuestras necesidades, no es algo sobre lo que realicemos una acción positiva y por tanto no es algo sobre lo que podamos tener o no razones para hacer. Sin embargo, desde los presupuestos de la psicología y desde otras posiciones se ha incluido a las necesidades entre las categorías motivadoras de la acción -deseos, impulsos, voliciones- para explicar a través de ellas el comportamiento. Así, la idea de necesidades ha servido para describir el conjunto de inclinaciones o potencialidades humanas que sugeriría la idea de motivación hacia algo; de ahí que se entienda como un concepto teleológico, orientado a objetivos, cercano al de deseo. ${ }^{13}$ Por otro lado, aun cuando frente a la noción de necesidades, las de deseos, voliciones $\mathrm{u}$ otras sean sentidas y articuladas subjetivamente, es cierto que pueden expresar necesidades y esto puede llevar a una nueva confusión terminológica en otro plano. A nuestro juicio, por el contrario, las necesidades juegan un papel de primer orden como razones justificativas. Precisamente éste es el punto de partida en el que nos apoyaremos para sostener que la función de la argumentación a través de las necesidades consistiría en aportar buenas razones para fundamentar los derechos.

Sin embargo, en el nivel de la vida cotidiana estas diferencias se desdibujan, ya que se utilizan las necesidades antes que los otros criterios porque todo el mundo sabe que no es lo mismo elegir o preferir algo apelando a la idea de necesidad que a las demás. El ejemplo más claro aparece en las campañas publicitarias que orientan el consumo. Incluso los niños saben que si piden algo diciendo que lo necesitan y no sólo que lo quieren está más justificado y nadie les negará algo que entienda que es necesario para ellos. La diferencia entre necesidades y deseos y la mayor fuerza argumentativa de aquellas vendría dada precisamente por su carácter objetivo, por tratarse de situaciones o estados de cosas que no dependerían de la intención del sujeto. Esa distinción se completaría por el criterio del resultado: el beneficio en el caso de los deseos, evitar un daño en el caso de las necesidades. Ciertamente, la idea de necesidad tiene una carga persuasiva importante que es utilizada por todos para justificar determinadas elecciones que entendemos que con la referencia a los deseos estarían débilmente apoyadas.

${ }^{13}$ P. Springborg, The Problem of Human Needs, cit., pág. 252. 


\section{Tipología de las necesidades}

Las diferentes aproximaciones sobre la noción de necesidades se plantean también con la existencia de diversos tipos de necesidades. Así, se habla de necesidades instrumentales que son relativas a determinados propósitos u objetivos ${ }^{14} \mathrm{o}$ entendidas como medios para perseguir preferencias particulares, frente a las necesidades básicas o fundamentales que son aquellas que no dependen de fines ni objetivos del sujeto, donde lo esencial se encuentra en el significado del término necesidad. También se distingue entre necesidades derivadas y no derivadas; ${ }^{15}$ las primeras son aquellas que tiene una persona en virtud de sus necesidades básicas, aunque éstas pueden ser igualmente básicas en determinadas circunstancias. Por ejemplo, cuando la única forma de obtener comida es comprándola, si una persona tiene necesidad de comer, tiene una necesidad derivada de dinero. Por el contrario, se tiene una necesidad no derivada cuando se necesita algo sin referencia a ninguna otra necesidad. Cabe una distinción ulterior entre necesidades constitutivas y necesidades circunstanciales. En el enunciado «A necesita $x$ en $\mathrm{C} », \mathrm{C}$ representa las circunstancias en las que A necesita $x$, por tanto, puede hablarse de necesidades relativas a esas especiales circunstancias. Serían necesidades constitutivas las que se dan en cualquier circunstancia.

En las diferentes aproximaciones teóricas sobre necesidades se ha distinguido también entre verdaderas y falsas necesidades, auténticas e irreales o necesidades manipuladas y no manipuladas. En concreto, lo que ha sido calificado como doctrina de las verdaderas y falsas necesidades surgió inicialmente de los intentos de mediación que configuraron el freudo-marxismo, que trataba de mostrar cómo la motivación de la conducta humana debía buscarse en ciertos impulsos, tendencias o rasgos referidos al hombre como ser físico con capacidades cognitivas e intelectuales; sin embargo el sistema social, en concreto el capitalismo, introyecta en los sujetos aquellas necesidades que entiende como imprescindibles para sobrevivir como sistema. En definitiva, el sistema controla al sujeto a través de sus necesidades. De ahí que Marcuse ${ }^{16}$ hable de falsas necesidades como aquellas creadas por intereses sociales que las imponen al individuo con el fin de reprimirlo.

\footnotetext{
${ }^{14}$ A. White, «Needs and Wants», art. cit., pág. 107.

${ }^{15}$ G. Thomson, Needs, cit. págs. 18-9.

${ }^{16}$ H. Marcuse, El hombre unidimensional, Barcelona, Ariel, 1984, pág. 27.
} 
Nielsen explica las falsas necesidades como aquellas situaciones u objetos que la gente quiere o desea como resultado de un estímulo social, un adoctrinamiento ideológico y que sin estos presupuestos no lo sentiría así. Es conocida la posición de Heller, ${ }^{17}$ a estos efectos, que niega toda posibilidad de hablar en términos de necesidades verdaderas y falsas a partir de un punto de vista ontológico sobre las mismas, pero que entiende posible un juicio ético sobre su bondad o maldad.

Obviamente, a la hora de establecer criterios de diferenciación aparece una nueva cuestión y es la referida al sujeto competente para decidir, por ejemplo, si los individuos y los grupos han de ir formulando las necesidades y sus prioridades o bien si se parte del presupuesto de que todas las situaciones que individualmente se sientan como necesidades deben ser reconocidas como tales. De esta forma, la solución a esta cuestión permitiría no sólo distinguir entre necesidades impuestas o no, sino también ofrecería un criterio importante a la hora de la argumentación de los derechos o si se prefiere a la hora de la argumentación sobre la fundamentación de los derechos.

\section{El carácter normativo}

A todo lo anterior se añade en el seno de las aportaciones sobre necesidades otra cuestión más que a nuestros efectos tiene una importancia bastante decisiva. Nos referimos al debate sobre el carácter normativo del término, que supone el planteamiento de cuestiones como las relaciones entre ser y deber ser o las relaciones entre la existencia de necesidades y exigencia de su satisfacción que tienen lugar al hilo de las consideraciones sobre las necesidades. Sobre este punto parece conveniente detenerse.

\section{EL CARÁCTER NORMATIVO DE LAS NECESIDADES Y LA RELACIÓN SER / DEBER SER EN EL CONCEPTO DE NECESIDADES}

Es cierto que la pregunta fundamental a la que tratamos de dar respuesta es, si las necesidades pueden encontrarse en la base de los derechos humanos, qué tipo de categoría o de concepto

\footnotetext{
${ }^{17}$ A. Heller, «Can true and false needs be posited?», The Power of Shame, London, Routledge and K. Paul, 1985, págs. 285-7.
} 
constituyen que posibilita justificarlos, ofrecer una adecuada fundamentación de los mismos y por qué. Esta pregunta que, insisto, es el objetivo básico de nuestra exposición, no sólo no encuentra una respuesta sencilla, sino que exige plantearnos como paso previo otro tipo de cuestiones que aparecen unidas a la idea de necesidad, básicamente la discusión en torno al carácter normativo de la idea de necesidades, a la separación-conjugación entre ser y deber ser en las tesis sobre necesidades.

En relación con ello, vamos a tratar de recoger algunas de las posiciones más destacadas del debate que tiene lugar en torno a esos dos aspectos en los que se plantea la discusión:

-Los elementos normativos y fácticos de la propia noción de necesidades.

-La relación entre necesidades y satisfacción de las mismas.

\section{Las tesis sobre la complicación entre hecho y valor en el concepto de necesidades}

Es preciso hablar en primer lugar de un grupo de posiciones que tendrían en común la complicación de hecho y valor en el concepto de necesidades partiendo de puntos de vista diferentes y concluyendo de ahí la objetividad de los valores y el vínculo indefectible entre necesidades, exigencias de su satisfacción, y derechos.

Una de las versiones que permite esta lectura consiste en afirmar que en las necesidades se produce una fusión entre hecho y valor. Es decir, que el conocimiento sobre las necesidades nos capacitaría, por ejemplo, en opinión de Maslow, ${ }^{18}$ para establecer valores que tienen una validez objetiva. Las consecuencias, es decir, el resultado, o el hecho mismo de que las necesidades resulten satisfechas, constituirían lo bueno y lo valioso. En este caso se encontrarían las necesidades psicológicas, las de seguridad, afecto, estima y de autorrealización o de experiencia plena.

En el mismo sentido se pronunciarían quienes describen la idea de necesidades a partir de un paradigma antropológico, quienes explican que las necesidades están relacionadas con una existencia valiosa y un desarrollo personal también valioso,

\footnotetext{
${ }^{18}$ A. Maslow, «Fusions of Facts and Values», American Journal of Psychoanalysis, vol. 23. 1961 pág. 120 .
} 
asumido como algo dado, como un a priori ontológico al modo del clásico proceso de transformación de la bellota al roble. (Fromm, Maslow, McClosckey). ${ }^{19}$

Desde un punto de vista relativamente distinto, otras posiciones (Ch. Taylor, McIntyre aunque desde presupuestos diferentes ${ }^{20}$ explican cómo en la idea de necesidad la noción de lo bueno o de valor no tiene lugar como algo separado absolutamente de los problemas de hecho. Es más, la apreciación de las necesidades provee las bases para evaluar algo como bueno, entendiendo por tal algo que no es una mera respuesta emotiva individual. Se califica así algo como bueno porque es objeto de nuestras necesidades y no al contrario, es decir, que algo es objeto de nuestras necesidades porque previamente ha sido entendido como algo valioso.

Es significativa, a estos efectos, la posición de Heller. En su construcción, las necesidades son entendidas como categorías antropológicas de valor. Es decir, las necesidades propiamente humanas son aquellas que desarrollándose a partir de las objetivaciones sociales son cualitativas, aquellas que desarrollan la «riqueza» humana, oponiéndose así a las necesidades alienadas. Las necesidades, por tanto, son afines a los valores, tanto a los propios de cada grupo social como a los valores de la humanidad, que se expresarían, entre otras formas, a través de las necesidades radicales que constituyen un ideal regulativo de una praxis orientada a la emancipación. ${ }^{21}$

La ventaja de la consideración de los valores como aquello que satisface necesidades sería que éstos no constituyen un sistema de criterios que se sobreponen a la realidad, sino que son una proyección de lo que el hombre hace de sí mismo, a través de su exteriorización, por tanto, según esta perspectiva, pueden ser entendidos como hechos operativos. Este argumento que parte de la constatación empírica de que el hombre tiene necesidades supone también que las necesidades tienden a satisfacerse

${ }^{19}$ H. J. McCloskey. «Human Needs, Rights and Political Values», American Philosophycal Quaterly, vol. 13, 1976, págs. 1, 6-8.

${ }^{20} \mathrm{Ch}$. Taylor, «Neutrality in political science». Philosophy, Politics and Society, Oxford, Basil Blackwell, 1969, dirigido por P. Laslett y W. G. Runcinman, págs. 25-57. A. MacIntyre, «Hume on is and ought», Against the SelfImages of the Age, London, Duckworth, págs. 109-124, esp. 120 y sigs.

${ }^{21}$ A. Heller, Hipótesis para una teoría marxista de las necesidades, Barcelona, Grijalbo, 1973, págs. 27-8. «Teoría, praxis y necesidades humanas», Teoría de las necesidades en Marx, cit. págs. 170-182. Por una Filosofia Radical, Barcelona, El viejo topo, 1980, págs. 110 y sigs. «Can true and false Needs Be Posited?», cit. 
inpendientemente de que tal satisfacción se obtenga realmente y que aquello que satisface una necesidad del hombre tiene un valor. El valor es, entonces, una abstracción mental entendida a partir de una experiencia concreta, la propia existencia de necesidades. Aquello que satisface una necesidad del hombre es un valor y lo que la contradice es un disvalor. ${ }^{22}$ En la misma línea, Marchello, ${ }^{23}$ señala que las necesidades implican los valores y éstos son inmanentes a la realidad de las necesidades. En cuanto las necesidades constituyen las raíces de las realidad práctica y en cuanto contienen implícitamente a los valores, sirven como criterio de interpretación y valoración de la praxis. El paso de las necesidades al valor no es problemático porque ambos coexisten en las necesidades. Estos criterios les sirven a los diferentes autores que hemos mencionado, para hablar de los valores como categoría objetiva, ya que éstos se justificarían en virtud de su idoneidad para satisfacer necesidades humanas.

En esta sede parece conveniente mencionar a quienes como Peters ${ }^{24}$ sostienen que las necesidades son un concepto fundamentalmente normativo en cuanto prescriben un conjunto de objetivos o estándares que implica que algo es malo o incorrecto con relación a una persona si en determinadas condiciones está ausente. Por ello la referencia a las necesidades significa un modelo que prescribe comportamientos, normas cuya ausencia debería dar lugar a una situación perjudicial o nociva para el sujeto.

¿Qué se consigue estableciendo el paralelismo, la coimplicación entre necesidades y valores a nuestros efectos? Por un lado, dar objetividad a los valores. Sin embargo, habría que hacer notar que en punto a la aportación de objetividad tal tesis adolece de la imprecisión derivada de no haber enunciado con claridad la categoría o tipo de necesidades a las que se refieren los valores que se toman en consideración. En segundo lugar, reconducir el problema de la fundamentación de los derechos al de los valores

${ }^{22}$ Este análisis fue realizado por N. Bobbio en su Introduzione alla filosofia del diritto, Giapichelli, Torino, 1948, pág. 35. Probablemente hoy no mantendría esta tesis, al menos, sin ulteriores especificaciones, pero la hemos recogido porque su exposición es un claro exponente de lo que aquí hemos querido señalar.

${ }^{23}$ G. Marchello, Dai bisogni ai valori. Nuovi Studi sull'eti dei dei valori, Torino, Giapichelli, 1977, págs. 35 y sigs.

${ }^{24}$ R. S. Peters, The concept of motivation, cit. págs. 17-8. En un sentido similar R. Dearden, «Needs in Education», Development of Reason, London, Routledge and K. Paul, 1972, págs. 50-64. 
y no permanecer en el terreno de las necesidades. ${ }^{25}$ En algunos casos, el recurso a las necesidades ha servido nada menos que para volver a encontrar los mismos problemas respecto a la fundamentación de los derechos humanos que plantea la justificación idealista de los mismos. Finalmente, se da como una evidencia que si existe una necesidad, ésta debe ser satisfecha y en consecuencia, según ello, existe un derecho a esa satisfacción. ${ }^{26}$ Esta ventaja constituye posiblemente el más grave de los inconvenientes: la confusión ontológica entre necesidades y derechos.

\section{Las dificultades de establecimiento del nexo entre ser y deber ser en las necesidades}

En el extremo opuesto encontramos a quienes sostienen que no cabe establecer un nexo causal entre la existencia de una necesidad y la exigencia de su satisfacción.

A estos efectos resulta importante retener la advertencia en la que insisten estos autores: la noción de necesidades puede ser utilizada fundamentalmente de dos formas: para hacer descripciones de situaciones de hecho o en sentido normativo.

El primer uso tiene lugar cuando se describen necesidades instrumentales o para referirse a situaciones en que las necesidades se asimilan a disposiciones connaturales, ${ }^{27}$ cuando se quiere expresar que alguien tiene un comportamiento motivado por una cierta disposición natural dominante. Las necesidades en este sentido son deseos o voliciones como motivos fuertes, es

${ }^{25}$ Quizá podría entenderse en este sentido la postura de uno de los primeros investigadores que entre nosotros ha tratado de poner en relación las necesidades básicas y los derechos humanos. Nos referimos a Pérez Luño que señala cómo a través de las necesidades puede articularse la relación ser-deber ser: «Esa articulación en lo que afecta al fundamento de los derechos humanos, se produce a medida que las necesidades trascienden el plano de los datos inmediatos sensoriales para devenir modos conscientes de preferencia, o sea, valores.» Derechos humanos, Estado de Derecho y Constitución, Madrid, Tecnos, 1984, pág. 184.

${ }^{26}$ En este sentido, por ejemplo, A. S. Kauffman identifica las exigencias de necesidades con las de derechos. Tener una necesidad es, por tanto, tener un derecho a ella. «Wants, Needs and Liberalism», Inquiry, vol. 14, 1971, pág. 194.

${ }^{27}$ En este sentido, P. Taylor explica que un comportamiento motivado por una disposición connatural dominante expresa que una persona tiene una tendencia a actuar de tal modo que da lugar a un resultado o a la realización de ciertos objetivos, incluso en circunstancias diferentes o cuando se dan disposiciones en conflicto. «Need Statements», Analysis, núms. 19-20, 1960, pág. 108. 
decir, que proveen razones para actuar en el sentido en que se hace.

El segundo uso es normativo. A partir de la noción de necesidad puede decirse que determinadas acciones han de ser realizadas, haciendo un juicio de valor respecto a lo que es mejor. Sólo en virtud del contexto en el que se formula la afirmación puede decirse si se utilizan las necesidades para hacer simples recomendaciones, una justificación, una explicación de la conducta o si se prescribe una acción en sentido fuerte.

Pues bien, según el punto de partida que adoptan los autores de esta posición, las descripciones sobre necesidades no proporcionan o no constituyen eo ipso una justificación para hacer prescripciones. ${ }^{28}$ Esto es, que si se quiere buscar un criterio que lleve a concluir o a decidir que algunas necesidades deben ser satisfechas, hay que fundamentar esta posición independientemente de las exigencias de necesidades, dado que es posible afirmar que los hombres necesitan determinadas cosas en el plano de los hechos y esto puede no ser deseable en el plano moral. En consecuencia, aun si pudiera mostrarse empíricamente que el hombre tiene determinadas necesidades básicas no sería, en opinión de Taylor, ni autocontradictorio ni lógicamente extraño abstenerse de recomendar su satisfacción o realización. La presunción de que una necesidad, una vez establecida empíricamente, debe ser satisfecha, se debe a la confusión entre esas exigencias y la utilización que se puede hacer de ellas con carácter normativo. La conclusión sería que entre ambas cuestiones no existen ni implicaciones lógicas ni contextuales.

Una versión parecida a ésta explica, como hace Fitzgerald, ${ }^{29}$ que para hablar con propiedad de necesidades sería imprescindible poder realizar tanto una teoría empírica sobre ellas como un análisis normativo que mostrara cómo pueden ser criterios de deber para la praxis. Ambas cosas serían imposibles:

1. Aun cuando en la realidad es un hecho que existe la relación entre hecho y valor, la noción de necesidades sólo aparentemente da la impresión de que traza un puente, de que partiendo del ser llega al deber ser.

2. Porque aun cuando contenga elementos de ambos tipos no provee el vínculo lógico para su realización. Es un error considerar que esta relación es lógica, racional, razonable o necesaria.

${ }^{28}$ Ibíd., págs. 110-111.

${ }^{29}$ R. Fitzgerald, «The ambiguity and rhetoric of Needs», Human Needs and politics, cit. págs. 203-204. 
Si se identifica lo valioso con la satisfacción de necesidades es por la carga emotiva y persuasiva del término.

3. Por tanto, la relación es problemática por la connotación emotiva del concepto y sobre ella no se puede decir nada racional. ${ }^{30}$

Desde un punto de vista relativamente distinto es representativa también la postura de White, que al intentar formular un concepto uniforme de necesidades, indentifica todas las necesidades con su acepción instrumental. Lo constitutivo del concepto de necesidad es su carácter instrumental. El enunciado que mejor expresaría este carácter: "A necesita $x$ en orden a $y . »$ En ese sentido existe una relación de necesidad «para algo». ${ }^{31}$ Esto significa, de un lado, que las necesidades no son categorías generalizables, ya que dependen de contextos dados. Por otro lado, es un error -en opinión de White- suponer que la noción de necesidad puede ser analizada en términos de razones para la acción». Las necesidades tampoco son normativas, ya que es simplemente una cuestión de hecho comprobar si existe un fin y si aquello que se necesita es la única forma de alcanzar tal objetivo.

Estas posiciones son significativas por cuanto ponen de manifiesto el aparente callejón sin salida de la teoría de las necesidades en punto a la relación entre la existencia de una necesidad y su exigencia de satisfacción.

\section{La relación entre existencia de necesidades y exigencia}

de satisfacción. Las necesidades como razones para la acción

Una tercera toma de postura trata de establecer las posibilidades de argumentar en favor de una exigencia de satisfacción de las necesidades humanas.

Esta posición acepta los puntos de partida de la anterior, esto es: a) que la existencia de una necesidad es una cuestión separada, diferente de si tales necesidades deben o no ser satisfechas, $\mathrm{y} b$ ) que entre ambas no cabe una relación lógica. Por tanto, su interés se dirige a señalar que en un contexto general,

\footnotetext{
${ }^{30}$ Ibíd., págs. 207-11.
}

${ }^{31}$ En el mismo sentido, A. Flew señala que si se necesita algo no es por sí mismo, sino como medio de satisfacción de alguna función, propósito, fin u objetivo ulterior. Por tanto, la pregunta clave en este punto es ¿para qué? «Wants, Needs, Choices or Commands», Human Politics, cit. pág. 216. 
una vez se ha mostrado la existencia de una necesidad, puede argumentarse que constituye una buena razón para su satisfacción; ${ }^{32}$ por ejemplo, si se afirma que los niños tienen necesidad de amor y afecto podemos decir que ésta es una buena razón para que se satisfaga la necesidad y que si mostramos que tal necesidad existe resultaría lógicamente extraño y autocontradictorio abstenerse de exigir su satisfacción. Si bien no cabe una relación de inferencia lógica entre ambos aspectos, la relación tampoco es meramente contingente o casual. A menos que se entendiera o se caracterizara una necesidad como destructiva habría que establecer una presunción general de que las necesidades constituyen un argumento suficiente para que aparezca un deber correlativo de satisfacción y justificar a su vez con razones por qué se niega la satisfacción a determinadas necesidades.

Las necesidades deben tener algún tipo de base que permita dar razones que expliquen o fundamenten su satisfacción. En este sentido, por ejemplo, no sería suficiente decir que alguien desea o quiere algo para afirmar que debe ser realizado; ciertamente los deseos también son motivaciones para la acción y sin embargo, su eficacia de justificación de la existencia de deberes correlativos dirigidos a satisfacerlos es cualitativamente distinta a la que pueden ofrecer las necesidades, como ya se ha visto.

Si hay un dato que haga que las necesidades constituyan un concepto especial, a diferencia de los deseos, intereses o aspiraciones, es que son insoslayables, lo que no comporta la noción de inalienabilidad. En este sentido el carácter definitorio de las necesidades no es que existan en función de fines y objetivos trazados por cada uno, sino que hacen referencia a situaciones cuya no satisfacción causa un perjuicio o un daño grave al sujeto o al grupo social. Con todo, el paso a su satisfacción no puede explicarse en términos prescriptivos.

En opinión de Thomson, que compartimos, las necesidades pueden constituir razones, pero no razones concluyentes. Ésta es una noción demasiado fuerte para atender, en términos generales al componente evaluativo de muchos conceptos normativos. Por ello, la noción «débil» de razón es el camino más adecuado para este análisis. Quizá valga la pena, pues, detenerse en esa noción de razón no concluyente. La tesis de Thomson vendría a mostrar que las razones no concluyentes o razones prima facie no pueden ponerse de relieve a través del análisis prescriptivo, ya

\footnotetext{
${ }^{32}$ Ésta es la posición de K. Nielsen, «On Human Needs and Moral Appraisals», Inquiry, 1965-66, pág. 170 .
} 
que son razones para la acción y por tanto no es adecuado un examen en términos de imperativos, ni en términos de principios prescriptivos, ni como imperativos condicionales.

En primer término trata de mostrar que la relación que existe entre una razón y una situación (hecho/is) podría ser diferente de las relaciones entre una razón concluyente, es decir, un deber y un enunciado de ser. Si se acepta la definición de razón concluyente como deber, de modo que la persona $\mathrm{P}$ tiene razones para hacer $\mathrm{A}$ en la situación $\mathrm{S}$ si y sólo si $\mathrm{P}$ tiene razones para hacer $\mathrm{A}$ y no existe una acción alternativa $\mathrm{B}$ en situación $\mathrm{S}$, tal que $\mathrm{P}$ tenga una mejor razón para hacer B que para hacer A, entonces existe una suerte de vacío entre afirmaciones de la forma «P necesita $\mathrm{X} » \mathrm{y}$ las razones concluyentes. Sin embargo, este vacío no es exactamente el mismo tipo de salto lógico que describen los prescriptivistas y la existencia de tal vacío es consecuente con que algunos hechos sean razones para la acción. Por tanto, desde el punto de vista prescriptivo no es posible hacer un análisis adecuado de las razones no concluyentes. ${ }^{33}$

En segundo lugar, cabe plantear si sería posible esta modificación a través de la consideración de las razones no concluyentes en términos de imperativos condicionales, de forma que el imperativo condicional pueda representar la idea de razón como regla que específica exactamente cuándo se tiene y cuando no una razón para hacer A. Así, cuando se expresa «puedo hacer A a menos que $\mathrm{C} 1, \mathrm{C} 2 \ldots \mathrm{Cn}$ ». Es decir, que la cláusula «a menos que» debe especificar completamente todas las situaciones posibles de manera que si alguna de ellas fuera cierta, entonces $\mathrm{P}$ tendría una razón concluyente para hacer A. Esto significa que si la serie $\mathrm{C}$ especificada en la cláusula fuera incompleta, podrían darse circunstancias al respecto y $\mathrm{P}$ tendría razones para hacer algo distinto de $\mathrm{A}$. La cuestión entonces estriba en que la serie $\mathrm{C}$ especificada debe ser completa y tal empresa no parece posible, al menos por dos razones: Por un lado, si se pretende hacer un listado, existe la imposibilidad de prever y dar razón de todas las posibles combinaciones de circunstancias que pueden formar tal regia. Por otro lado, si se opta por no hacer descripciones específicas, sino generales, éstas no podrían comportar reglas cuya aplicabilidad sea establecida por la misma regla. La conclusión que de aquí se extrae es que esta segunda vía, la de los imperativos condicionales, no puede tampoco utilizarse para analizar la noción de razón como razón prima facie. $^{34}$

\footnotetext{
${ }^{33}$ G. Thomson, Needs, cit. págs. 114-15.

${ }^{34}$ Íbid., pág. 117.
} 
Una de las ventajas de las razones no concluyentes es que pueden actuar en un número indefinido de contextos, ya que independientemente del contexto no es factible esperar que se especifique cuándo alguien tiene una razón concluyente o no para una acción de determinado tipo. Sin embargo, la noción de razón concluyente es demasiado fuerte para captar en términos generales el componente evaluativo de muchos conceptos normativos. Las necesidades como razones para la acción exigen poder predicar de ellas determinadas características:

La primera, que estas razones pueden ser obviadas en ciertos casos. Lo que exige, por un lado, su no consideración como excepciones a un principio y, por otro, que para obviar una razón porque no parece adecuada, hay que tener, a su vez, razones. Si fueran consideradas como excepciones a un principio prima facie, tal como las entiende Hare, ${ }^{35} \mathrm{se}$ podrían proponer como algo casual y menos razonable que el principio y vaciarlo de contenido sin razones suficientes. Por ello, Thomson indica que los principios prima facie tienen la forma «hay una razón para hacer A» o «hay una razón para no hacer $A$ » tanto, que estos principios deberían ser analizados en términos de una razón para la acción. Es decir, que sería posible obviar un principio en un determinado contexto y no por ello se haría una excepción a tal principio.

La segunda característica reside en la posibilidad de poder argumentar, a este respecto, sobre aquellas razones que parecen mejores o más fuertes. Es decir, que unas razones pueden superar a otras. Esta apreciación puede servir, por ejemplo, para argumentar por qué las necesidades constituyen mejores razones que los deseos, aunque éstos también sean razones para la acción intencional, en algunos casos.

La tercera consiste en rechazar las tesis prescriptivas para explicar el concepto. Así, lo que en el terreno de la razón no concluyente parece preciso recuperar es la noción de una razón que sea posible obviar para poder hacer afirmaciones tales como «las promesas crean obligaciones»o «las necesidades proveen razones para la acción». ${ }^{36}$

En definitiva, tener una necesidad puede ser entendido como «hay una razón para hacer A», lo que no significa una prescripción. Las necesidades aportan argumentos sobre aquellas razones que parecen mejores o más fuertes. Por otro lado, la determinación

\footnotetext{
${ }^{35}$ Hare, Moral Thinking, Oxford, Clarendon, 1981, pág. 59.

${ }^{36}$ G. Thomson, Needs, cit. pág. 122.
} 
del concepto no puede quedar limitada a la capacidad de cada persona para dar razones de sus propias necesidades, sino que éstas tienen algún aspecto de carácter objetivo.

De lo anterior se sigue, en nuestra opinión, la conveniencia de situar el planteamiento de las necesidades en el ámbito de la racionalidad discursiva, esto es, en aquel ámbito que permite dar razón de por qué se realizan determinadas acciones o por qué se toman ciertas decisiones. Lo que significa que, en definitiva, el discurso sobre las necesidades nos conduce a los criterios de razonabilidad y racionalidad.

Tomemos las dos acepciones del concepto de necesidad que nos parecen más relevantes: necesidades instrumentales, aquellas que lo son en virtud de un fin u objetivo, y necesidades fundamentales que se referirían a aquellas que evitan un perjuicio o daño grave a la persona, de acuerdo con las condiciones señaladas. Veamos si cabe hablar de racionalidad respecto a ellas. El requisito fundamental para hablar de racionalidad respecto a la acción es, tal como lo señala MacCormick, ${ }^{37}$ que cada acto u omisión debe estar justificado con referencia a alguna razón para la acción. Es decir, un acto ha de ser entendido como racional si el sujeto tiene alguna razón para hacerlo y lo realiza por tal razón, del mismo modo que la racionalidad particular de un acto en el tiempo está condicionada por su pertenencia a un modelo de actividad «sobre» el tiempo que es en sí mismo racional. Sin embargo, son exigibles otros órdenes de razones para evaluar las de primer orden: las razones para la acción, y éstas exigen consistencia y coherencia. Asimismo, decimos que las decisiones son racionales cuando son universalizables, aptas para que cualquier persona las pueda adoptar. Cabe también que se pueda predicar de tales decisiones la cualidad sistemática del discurso. De nuevo con MacCormick, la racionalidad en la acción es algo más que tener simplemente algunas razones para realizar un determinado acto. Tanto respecto a la esfera de la razón práctica como a la especulativa son necesarias razones de segundo orden para discriminar entre las de primer orden.$^{38}$ En definitiva, se trata de poner de relieve que para afirmar que una acción es racional no basta, aun cuando a efectos explicativos haya sido el punto de partida, decir que se tienen razones para

\footnotetext{
${ }^{37}$ N. MacCormick, «Limits of Rationality in Legal Reasoning», An Institutional Theory, Dordrecht, Reidel Publ., 1986, pág. 190.

${ }^{38}$ N. MacCorrnick, Legal Reasoning and Legal Theory, Oxford, Clarendon, 1978, págs. 266 y sigs.
} 
actuar, sino que tales razones precisan reunir una serie de caracteres determinados.

Sin embargo, aun cuando las decisiones hayan sido adoptadas siguiendo las exigencias del razonamiento práctico, es posible llegar a una conclusión racional pero no razonable. Como pone de relieve Aarnio ${ }^{39}$ respecto al razonamiento jurídico, en cuanto razonamiento práctico, el discurso racional ofrece ciertas garantías para la justificación del razonamiento, pero no puede asegurar totalmente su legitimidad, esto es, la aceptabilidad del mismo. Lo que se pretende es que el modelo discursivo racional se construya como ideal a partir del cual se pueda evaluar tal legitimidad.

Hasta aquí podemos afirmar que las necesidades pueden entenderse como razones prima facie para la acción y de este modo podrían entenderse como hechos que por sí mismos, según la definición de Raz, ${ }^{40}$ bastan para determinar la acción con una cierta orientación, siempre que no haya otros factores que muestren lo contrario. Ahora bien, volviendo a un argumento que ya hemos mencionado anteriormente, las necesidades no sólo constituyen razones para la acción, sino que una vez establecida una necesidad ésta es por sí misma buena razón para que sea satisfecha. Éste es el dato clave que permite hablar de las necesidades no como razones débiles sino fuertes, porque son razones suficientes o buenas razones. Acudiendo de nuevo a MacCormick, ${ }^{41}$ una buena razón es una razón suficiente para la acción que exige, a su vez, razones evaluadoras o criterios de evaluación, a partir de los cuales es posible «preferir» unas razones frente a otras en contextos dados. A este respecto indica, como cuestión previa, cómo el tipo más simple de razón para hacer algo se encuentra en la apelación al deseo de hacerlo o como consecuencia de la satisfacción que obtendrá el sujeto por su realización, pero que los deseos, pasiones, afectos, emociones, etc., en la medida en que configuran los propósitos más primitivos -lógicamente- no son racionales ni irracionales, sino simplemente no racionales. Por ello, resulta imprescindible que los propósitos e intenciones que se trata de llevar a cabo estén sujetos a la disciplina de algún tipo de racionalidad, que puedan establecerse como órdenes de preferencia racionales, de forma que sea posible

${ }^{39}$ A. Aarnio, «Sobre la racionalidad. Algunas observaciones sobre la racionalidad jurídica», ACFS, 1983-4, pág. 17.

${ }^{40}$ J. Raz, Razonamiento práctico, México, FCE, 1978, pág. 29.

${ }^{41}$ N. MacCormick, An Institutional Theory of Law, cit. pág. 194. 
establecer un cierto conjunto de fines, objetivos o propósitos que se determinen a partir de la generalización de los propósitos reales y actuales y cuya realización se considere admisible. Es decir, que en un sistema así establecido, no sería aceptable una razón como buena o suficiente a menos que pudiéramos realizar tales objetivos a través de acciones que en el sistema estén calificadas como buenas o en cualquier caso no erróneas. De manera que estas buenas razones lo serían tanto para guiar la conducta humana como para formularse como principios generales de acción.

De todo lo anterior cabe formular tres cuestiones que deben ser sometidas a debate:

1. Las necesidades constituyen razones para realizar determinados fines, guiar conductas, orientar acciones, etc.

2. Las propias necesidades comportan o pueden dar lugar a buenas razones o razones suficientes para exigir su satisfacción.

3. La exigencia de satisfacción de las necesidades humanas básicas ¿es una razón suficiente para su reconocimiento como derechos? Sobre esta cuestión es sobre la que hablaremos en lo que sigue.

\section{SOBRE LA POSIBLE FUNDAMENTACIÓN DE LOS DERECHOS HUMANOS DESDE LAS NECESIDADES BÁSICAS}

Trataremos de formular nuestra propuesta de fundamentación a través de una serie de argumentos que en realidad se dirigen a presentar problemas abiertos que deben ser sometidos a discusión. Tales argumentos nos conducirán a enunciar una conclusión poco concluyente: que la noción de necesidades humanas básicas nos ofrece argumentos nada desdeñables para clarificar, junto con otros formulados desde diferentes perspectivas, el problema abierto de la fundamentación, o, dicho en otros términos, que la teoría de las necesidades en relación con los derechos humanos es útil sobre todo a la hora de ofrecer argumentos de fundamentación de los derechos, y no para establecer directamente la existencia de los mismos. En este sentido y desde los intentos que hasta ahora se han propuesto de una posible fundamentación de los derechos humanos en las necesidades básicas podemos afirmar que el recurso a las necesidades supondría aceptar que en ellas se encuentra el sustrato antropológico de los derechos, de forma que reconocer, ejercer y proteger un derecho básico significa, en última instancia, que se pretende satisfacer una serie de necesidades, entendidas como exigencias de una 
vida digna. ${ }^{42}$ De este modo se explica la objetividad y el carácter generalizable del fundamento, que, por otro lado, supera también el reduccionismo propio de la apelación a los deseos o intereses de los individuos que daría lugar a un objetivismo extremo. En segundo lugar, las necesidades constituirían a la vez el contenido de valor que informa tales derechos, valores que por su inserción en la experiencia histórica de las necesidades sociales no configuran un sistema cerrado y estático de principios absolutos situados en una esfera anterior e independiente de la experiencia humana. Desde una perspectiva diferente hay que anotar la propuesta de Hierro en el que, posiblemente, es el primer trabajo publicado entre nosotros sobre el aspecto concreto que ahora nos ocupa ${ }^{43}$ y en el que se mantiene el recurso a la noción de necesidades como elemento de posible objetivación de la base misma del concepto de derecho.

Efectivamente, la posibilidad de que las necesidades constituyan el fundamento o la base de los derechos puede ser formulada desde dos puntos de vista diferentes: 1) Según el primero, un derecho tiene lugar a partir de una necesidad básica porque se entiende que existe una conexión directa entre ambos. La necesidad va dirigida a algo que se considera valioso, las necesidades y los valores son afines y el derecho no se funda directamente en la necesidad, que sería un soporte ulterior, sino en el valor al que dan lugar y por lo que existe una necesidad «en orden a»; 2) la segunda versión estima que entre necesidades y derechos hay una relación mediada por la «prueba» de una exigencia fuerte (claim), donde las necesidades apoyarían, argumentarían a favor de un tipo de pretensión que en determinados supuestos puede traducirse en un derecho. Este último planteamiento exige no confundir necesidades como razones para la existencia de un derecho con la necesidad como derecho.

Por nuestra parte trataremos de exponer brevemente en forma de cinco tesis nuestras propuestas sobre el particular:

Primera.- La función de las necesidades básicas se cumple en la esfera de la fundamentación de los derechos humanos más que respecto a la existencia de los mismos. A la vista de lo anterior, creemos poder concluir la imposibilidad de construir una teoría de las necesidades cuya proyección sobre la teoría de los

${ }^{42}$ En este sentido, puede verse A. E. Pérez Luño, Derechos humanos, cit. págs. 176-184. E. Fernández, Teoría de la justicia y derechos humanos, Madrid, Debate, 1984, págs. 78-9.

${ }^{43}$ L. Hierro, «¿Derechos humanos o necesidades humanas? Problemas de un concepto», Sistema, núm. 46, 1982, págs. 45-61, esp. 53 y sigs. 
derechos humanos resuelva todos los problemas relativos a la fundamentación y a fortiori, los problemas de técnica jurídica que todo ello comporta. Sin embargo, la teoría de las necesidades ofrece argumentos muy válidos para clarificar, junto con otros, el problema abierto de la fundamentación. Por lo demás, entendemos que responde a lo que sería una adecuada fundamentación, que no puede sino significar la formulación de criterios y presupuestos racionales y razonables que puedan contribuir a justificar los derechos humanos. Por tanto, el fundamento que proponemos no se presenta como absoluto, porque no se trata de reducir la multiplicidad de lo real a un único factor que determine el resto de elementos que de él derivarían y, por otro lado, habría que matizar que el hecho de reconocer la pluralidad de determinaciones y objetivaciones sociales y los diferentes criterios explicativos, no supone rechazar la búsqueda de algún tipo de fundamentación.

Segunda. -Independencia entre existencia y satisfacción de las necesidades. Entendida esta última como la esfera propia de los derechos (los derechos como exigencia de satisfacción de las necesidades). Primero, porque establecer la relación entre necesidades y valores no comporta la relación directa entre necesidades y valores jurídicos. En segundo lugar, porque el lenguaje de las necesidades es mucho más amplio que el de los derechos. Por tanto, habría que distinguir entre aquellas necesidades que pueden ser especificadas en términos de derechos y aquellas que no pueden serlo. El ejemplo básico es la necesidad de amor y afecto. No todas las necesidades supondrían derechos, porque la exigencia de su satisfacción no siempre capacita al individuo para exigirlas como tales. De modo que no todas las necesidades tienen una traslación directa al terreno de los derechos. En realidad, esta tesis se articula a través de las siguientes afirmaciones básicas:

a) Como hemos señalado, los enunciados «X necesita $\mathrm{Y}$ para $\mathrm{Z} »{ }^{44} \mathrm{y}$ «X puede exigir la satisfacción de $Y »$ expresan cuestiones diferentes cuya relación no es directa ni puede configurarse como una inferencia lógica.

b) Afirmar que las necesidades pueden constituir, en su calidad de buenas razones, fundamentación de derechos no significa que podamos conceptuar a los derechos como necesidades ni aun como necesidades básicas. Efectivamente, aunque se trate de

\footnotetext{
${ }^{44}$ A los efectos de la presente argumentación prescindimos de la discusión teórica acerca de la relevancia de la cláusula «en orden a $Z$ » $\mathrm{o}$ «para $Z$ » a la hora de la formulación del concepto mismo de necesidades.
} 
cuestiones estrechamente implicadas tampoco cabe identificar concepto y fundamentación de derechos. ${ }^{45}$

c) Por otro lado, el hecho de que podamos argumentar razonablemente que las necesidades humanas básicas deben ser satisfechas no comporta que exista un derecho como tal, directamente inferido de la anterior afirmación y atribuido a los titulares de las necesidades.

d) Todo lo anterior no significa que no se pueda argumentar concretamente y queremos decir con ello argumentar de acuerdo con los parámetros de razonabilidad, de manera tal que desde la existencia de necesidades podamos llegar a la existencia de derechos. Precisamente cuando se establece la vinculación entre necesidades y derechos prima facie se está haciendo referencia al tipo de exigencias que comportan las necesidades que son distintas -por ser fuertes- de las exigencias propias de deseos, voliciones, aspiraciones, etc.

Tercera. -Carácter objetivable de las necesidades. A nuestro juicio es posible llegar a establecer criterios que permitan discernir cuándo estamos en presencia de una necesidad humana básica, de acuerdo con alguna de las aportaciones aquí examinadas. Efectivamente, cuando podemos razonar y analizar una situación tal que su no satisfacción causa un daño o perjuicio, no cualquier perjuicio o daño, sino el que podemos calificar como grave, tanto en orden a la supervivencia como al desarrollo de la persona como tal. Esto comporta, al menos, abordar los siguientes problemas:

a) En primer lugar, conseguir explicar por qué es malo carecer de algo o, por decirlo mejor, es necesario dar razones que prueben que en determinadas situaciones se produce directamente un perjuicio, un detrimento personal si no se satisface esa necesidad. Es decir, que tal perjuicio permanecerá y con la misma gravedad a menos que la necesidad en cuestión resulte satisfecha y que no hay forma de suplir esa carencia sino satisfaciendo la necesidad; no hay por tanto alternativas. Por supuesto que esto comporta también advertir que no toda privación o carencia tiene idénticas consecuencias, del mismo modo que no toda frustración de un deseo produce un perjuicio. Se hace preciso atender a los tipos generales de experiencias, decisiones y acciones de la persona y no a contratiempos o privaciones pasajeras o que

\footnotetext{
${ }^{45}$ Sobre la importancia de la distinción de ambos planos, véase F. Laporta, «Sobre el concepto de derechos humanos», Doxa, núm. 4, 1987, págs. 23-46.
} 
pueden tener satisfacciones sustitutivas para determinar el contenido objetivo del daño. ${ }^{46}$

b) Efectivamente, la ausencia de bienes primarios constituye un perjuicio a la persona al igual que la de otras situaciones y bienes que no siendo primarios son básicos. Pero lo decisivo aquí es argumentarlo de manera que reciba esa consideración y que sea aceptado como tal perjuicio por parte de la comunidad. Esto supone, de una parte, dar razones del daño que produce esa carencia $\mathrm{y}$, de otra, justificar asimismo por qué, en los supuestos concretos, determinadas carencias no deben ser satisfechas. A este respecto nos parece de enorme utilidad interpretativa el carácter de inescapability con el que Thomson define el tipo de necesidades cuya satisfacción debe alcanzar el rango normativo en cuestión.

En definitiva, con todo ello no tratamos sino de concretar una afirmación básica que obteníamos ya desde el principio: las dificultades de determinación positiva del concepto y el contenido de las necesidades humanas, incluidas las básicas.

Cuarta.- El carácter histórico de las necesidades. Éste constituye otro aspecto que resulta imprescindible señalar, ya que aun admitida la posibilidad de objetivación de las necesidades, tal como la hemos expuesto, no podemos perder de vista que tanto las condiciones en las que aparece la conciencia sobre las necesidades, como las exigencias de satisfacción de las mismas $\mathrm{y}$, a fortiori las formas de satisfacción, son históricas. Precisamente, esa historicidad tiene como consecuencias respecto a la posibilidad de fundamentar los derechos desde las necesidades:

a) Las condiciones que de hecho permiten que surja la conciencia de las necesidades que hace posible su reconocimiento y exige su satisfacción en forma de derechos son históricas, ${ }^{47}$ lo que quiere decir, en nuestra opinión, que obviamente no existe un catálogo cerrado de las necesidades, ni siquiera por lo que se refiere a las necesidades básicas; de otro modo volveríamos a las construcciones que presentan uno u otro modelo de naturaleza humana universal e inmutable, esto es, al paradigma clásico de

\footnotetext{
${ }^{46}$ Respecto al análisis del carácter objetivo de la noción de daño como elemento constitutivo de la noción de necesidades puede verse G. Thomson, Needs, cit. págs. 35-63.

${ }^{47}$ Sobre el carácter histórico no tanto de las necesidades en sí mismas, sino sobre todo de las circunstancias a partir de las cuales pueden ser traducidas como derechos fundamentales, véase L. Prieto, «Ideología liberal y fundamentación iusnaturalista de los derechos humanos. Observaciones críticas», Anuario de derechos humanos, núm. 4, 1986-87, págs. 291-321, esp. pág. 314.
} 
la fundamentación iusnaturalista de los derechos humanos. Correlativamente, en la medida en que las necesidades constituyen razones que están en la base de los derechos, el catálogo de los mismos no puede ser cerrado, precisamente porque su propia concepción exige recoger como derechos aquellas exigencias que se consideran insoslayables porque las apoyan tales «buenas razones».

b) Por otro lado, reconocer que tanto las mismas exigencias de satisfacción de las necesidades como las modalidades con las que se puede responder a dichas pretensiones sean históricas, según es evidente, permite un acercamiento más adecuado a la fundamentación de los llamados derechos económicos, sociales y culturales y por extensión a los derechos de la tercera generación. Así se ponen de relieve las posibilidades que se abren si se conectan necesidades humanas básicas, derechos económicos y sociales y programas de desarrollo, entendiendo por tal el desarrollo de los seres humanos, lo que exige, y esto nos parece algo de gran trascendencia, cambiar los parámetros para medir estos procesos $\mathrm{Y}$ por tanto, no atender tanto -como criterio fundamental- a la riqueza que se produce, sino a la miseria y a las situaciones de los más desfavorecidos. ${ }^{48}$ En efecto, la fundamentación de estos derechos no resulta fácil desde algunas de las teorías que tratan de dar respuesta al problema general de la fundamentación de los derechos característicos de la concepción liberal individualista, como ya hemos explicado. Por el contrario, desde las necesidades entendidas como buenas razones, puede ofrecerse no sólo una fundamentación más adecuada a estas categorías de derechos, sino también una respuesta que supere el problema del titular de las correlativas obligaciones. Obviamente, todo ello está estrechamente relacionado con la última de nuestras tesis.

Quinta.- Las necesidades constituyen razones para la acción. Como ya hemos advertido en la segunda tesis, el carácter normativo de las necesidades no agota su concepto ni significa una

${ }^{48}$ Como es sabido los programas de desarrollo económico fueron planteados a partir de la idea de necesidades básicas, sobre ello, por ejemplo: P. T. Muchlinski, «Basic Needs Theory and Development Law», International Law if Development: Comparative Perspectives, Abingdon, Professional Books Limited, dirigido por F. Snyder y P. Slim, 1987, págs. 237-270. I. M. Samater, «De crecimiento a necesidades básicas, la evolución de la teoría del desarrollo», Artículos de la MONTHLY REVIEW, Madrid, Revolución, 1986, págs. 71-85, J. McHale y M. C. McHale, Basic Human Needs, New Jersey, Transaction Books, 1977. 
dimensión prescriptiva en sentido estricto. La idea de necesidad recoge, efectivamente, aspectos múltiples y por tanto diferentes funciones, por ejemplo, motivaciones para la acción, forma de relación del hombre con el mundo en el que vive, principio explicativo de la socialidad e incluso, desde otros presupuestos, principios regulativos, pero desde el punto de vista que aquí sostenemos, constituyen sobre todo una razón para la acción o, mejor dicho, razones. Sin embargo, no todas las necesidades, como venimos afirmando, cristalizan en derechos al no poder constituir buenas razones o razones suficientes para entender que exigen un tipo de reconocimiento, de protección y garantías que sólo el Derecho puede dar. Por ello, como explicábamos con Raz, MacCormick, Aarnio y Thomson, hay que acudir al criterio de racionalidad y razonabilidad para su traslación al ámbito de los derechos, proceso que pasa por el consenso de la comunidad para su aceptabilidad y legitimación pero que exige además un aparato discursivo racional previo. De acuerdo con ellos y tal como recientemente entre nosotros ha señalado Atienza-, ${ }^{49}$ no utilizamos razonabilidad en un sentido amplio, sino de un modo que trata de ser muy preciso, que supone previamente, como decimos, un aparato discursivo racional, pero que apela sobre todo a las nociones de equilibrio entre las pretensiones enfrentadas y consenso. Indudablemente el problema de las necesidades es, y ello constituye una de nuestras conclusiones, sobre todo un problema de interpretación. Pues bien, precisamente en el terreno de la interpretación -por ejemplo, y muy especialmente respecto al problema de la igualdad material-, en el terreno de la argumentación jurídica y más concretamente de la argumentación judicial, con referencia a la Constitución, es donde pueden jugar las necesidades, en su relación con los derechos constitucionales y, es ahí, desde luego, donde los elementos de la noción de razonabilidad parecen especialmente adecuados al propio concepto de necesidades.

${ }^{49}$ M. Atienza. «Para una razonable definición de «razonable», DOXA, núm. 4, 1987, págs. 189-200. También J. de Lucas y E. Vidal, «Razonabilidad e interpretación constitucional: a propósito de una jurisprudencia reciente», Dirección General de lo Contencioso del Estado, Madrid. 1989 (en prensa). 